\title{
Bolometric Response of Optically Thick Superconducting Films
}

\author{
J.D. Chem and H.C. Yang \\ Department of Physics, National Taiwan University, Taipei, Taiwan, R.O.C. \\ Z.D. Genchev \\ Institute of Electronics, Bulgaria Academia Sinica, 1784 Sofia, Bulgaria \\ H.E. Horng \\ Department of Physics, National Taiwan Normal University, Taipei, Taiwan, R.O.C. \\ D. Dew-Hughes \\ Department of Engineering Science, University of Oxford, Oxford OX1 3PJ, U.K.
}

\begin{abstract}
A one-dimensional heat conduction model is established to interpret the chopping frequency dependence of the bolometric response of optically thick superconducting films. The key feature of this model is that it takes into account the thermal boundary conductance of both the film/substrate and the substrate/lueat-sink interfaces. The model is found to be suitable for both the strong and weak thermal coupling cases. By comparing the results with a simplified bolometric model, explicit expressions of the thermal conductance, thermal capacitance, radiation absorption efficiency and response time constant of the bolometric system have been deduced, where the influences of the film, substrate and the heat conduction boundaries are all considered.
\end{abstract}

\section{INTRODUCTION}

With the employment of a lock-in amplifier and a four-probe measurement configuration, operated at a constant bias current, $I$, and at a stabilized temperature, $T$, around the midpoint of the superconducting transition, the dependence of the bolometric optical response, $V_{o r}$, of a superconducting film on the chopping frequency, $f$, usually decreases monotonically as the chopping frequency is increased [1]. A proportional relation between $V_{o r}$ and $f^{-\alpha}$ is presumed to study the dependence of $V_{\text {or }}$ vs. $f$, where the parameter $\alpha$ can be determined experimentally by the slope of the logarithmic plot of $V_{\text {or }}$ vs. $f$ characteristics. Various values of $\alpha$ between $\theta$ and $l$ have been reported. Dwir and Pavuna observed the optical response of $\mathrm{YBCO}$ films and concluded that the parameter $\alpha$ equals 0.5 for strong thermal coupling cases, and equals $l$ for weak thermal coupling cases, where two different models were used to interpret the observed experimental results [2]. However, other $\alpha$ values of $0.4[3]$, and 0.47 [4] were reported, not quite consistent with the predictions given by the aforementioned models [2]. The widely-spread $\alpha$ values indicate that the heat conduction behavior of the system

Manuscript received August 26, 1996

This work is supported in part by the National Science Council of R.O.C. under Grant No. NSC 85-2811-M002-011 and NSC 85-2112-M002-020. J.D. Chern was supported by both the John Swire and Sons Limited, and the Overseas Research Studentship in Britain. Z.D. Genchev was supported by the Bulgarian Ministry of Science under grant $\mathrm{Ph}-442$. is more complicated than the previous suggestions of Dwir and Pavuna. Based on the previous work of Genchev et al. [5], a more general heat-conduction model is, thus, required to sort out the inconsistency.

\section{MODELING}

\section{A. Radiation Absorption of the Film}

A simplified one-dimensional model of volumetric radiation absorption is used to estimate the absorbed radiation power density of a superconducting film (thickness $d_{f}$ ). Consider the case that a helium-neon laser perpendicularly irradiates the surface of an optically thick superconducting film. Since the radiation intensity decreases rapidly as the light propagates into the film, the reflected radiation intensity by the film/substrate interface is, thus, negligible.

The origin of the coordinate is chosen to be at the center of the radiation spot, assumed to be aligned to the point at the center position of the superconducting pattern. The electric field, $\vec{E}$, of the radiation is assumed to oscillate along the $\mathrm{x}$ direction at the radiation angular frequency, $\omega_{r}$, expressed by $\vec{E}=E_{x}(z) \cdot \exp (-i \omega r \cdot t) \hat{x}$, and the magnetic field, $\vec{H}$, along the $y$-direction, expressed by $\vec{H}=H_{y}(z) \cdot \exp (-i \omega r \cdot 1) \hat{y}$. The $z$-direction and $y$-direction are shown in Fig. 1. The derivation is based on the following Maxwell equations

$$
\begin{aligned}
\vec{\nabla} \times \vec{E} & =-\frac{\partial \vec{B}}{\partial t}, \\
\vec{\nabla} \times \vec{H} & =\vec{J}+\frac{\partial \vec{D}}{\partial t},
\end{aligned}
$$

where $\vec{B}, \vec{D}$, and $\vec{J}$ are the magnetic induction of the radiation, the electric displacement of the radiation, and the free current density caused by charged particles, respectively.

The relation between $\vec{D}$ and $\vec{E}$ fields, and between $\vec{B}$ and $\vec{H}$ fields are expressed by

$$
\vec{D}=\varepsilon_{0} \cdot \varepsilon_{r} \cdot \vec{E}
$$




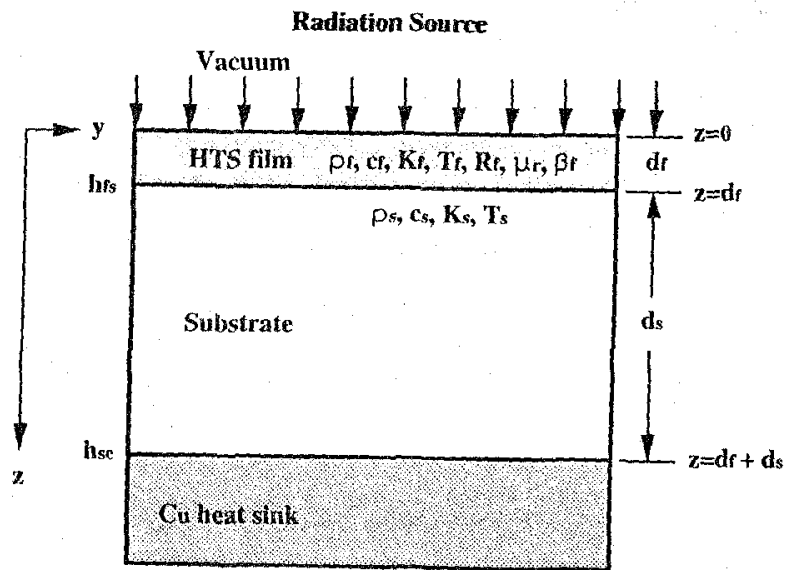

Fig. 1. A schematic diagram of the modified heat-conduction system, consisted of a superconducting film, a substrate, and a copper heat-sink.

$$
\vec{B}=\mu_{0} \cdot \mu_{r} \cdot \vec{H}
$$

where $\varepsilon_{r}$ are the relative permittivity of the film and $\mu_{r}$ the relative permeability of the film. Since the interested operation temperature is limited to a relatively small range around the midpoint of the superconducting transition, a finite conductance of the film is presumed, and the relation between $\vec{J}$ and $\vec{E}$ of the superconducting medium is assumed to obey the Ohm's law

$$
\vec{J}=\sigma \cdot \vec{E}
$$

where $\sigma$ is the electric conductivity of the film at the laser radiation frequency.

The one-dimensional volumetric radiation absorption of the film is based on the dissipation of average radiation power density, $\left\langle Q_{f}(z)\right\rangle$, absorbed by the film as given by

$$
\left\langle Q_{f}(z)\right\rangle=\frac{1}{2} \cdot \operatorname{Re}\left(\vec{J} \cdot \vec{E}^{*}\right) .
$$

Therefore, in the range of $0<z<d_{f},\left\langle Q_{f}(z)\right\rangle$ can be expressed by

$$
\left\langle Q_{f}(z)\right\rangle=J_{0} \cdot\left(\frac{1-R_{f}}{\mu_{r}}\right) \cdot \beta_{f} \cdot \exp \left(-\beta_{f} \cdot z\right),
$$

where $I_{0}$ is the output intensity of the laser, $R_{f}$ is the reflectance of the film, $\beta_{f} \equiv 1 / \alpha_{f}$ is the absorption coefficient of the film, and $\alpha_{f}$ the optical penetration depth of the film.

When the attenuation effects of the laser power density caused by an optical window and an optical chopper are taken into account, the sinusoidal component of the modulated laser power density in the film, oscillated at the chopping frequency, $f$, can be expressed by

$$
\left\langle Q_{f}(z, t)\right\rangle=\left\langle Q_{f}(z)\right\rangle \cdot \tau_{w} \cdot \tau_{c} \cdot \cos (2 \pi \cdot f \cdot t),
$$

where $\tau_{w}$ is the transmittance of the window, and $\tau_{c}$ the transmission factor of the chopper. For an idea square-wave- modulated laser beam, the transmission factor, $\tau_{c}$, is given by a constant value of $2 / \pi[6]$. The quantity $\left\langle Q_{f}(z)\right\rangle$ in $(7)$ is only valid for optically thick films, restricted by $d_{f}>\alpha_{f}$.

\section{B. Heat Conduction Process}

After the absorption of laser energy of the film, the absorbed heat is transferred by conduction down to the film, the substrate, and the heat-sink. The heat conduction equations [7] of the film and the substrate are given by

$$
\begin{gathered}
K_{f} \cdot \frac{\partial^{2} T_{f}(z, t)}{\partial z^{2}}-\rho_{f} \cdot c_{f} \cdot \frac{\partial T_{f}(z, t)}{\partial t} \\
=-\left\langle Q_{f}(z, t)\right\rangle, 0 \leq z \leq d_{f}
\end{gathered}
$$

where $T_{f}$ and $T_{s}$ are the sinusoidal temperature rise of the film and of the substrate, respectively, $K_{f}$ the thermal conductivity of the film, $\rho_{f}$ the mass density of the film, $c_{f}$ the specific heat of the film, $K_{s}$ the thermal conductivity of the substrate, $\rho_{s}$ the mass density of the substrate, and $c_{s}$ the specific heat of the substrate. The boundary conditions at the interfaces are given by

$$
\frac{\partial T_{f}}{\partial z}=0, \text { at } z=0
$$

$$
\begin{gathered}
K_{f} \cdot \frac{\partial T_{f}}{\partial z}=K_{s} \cdot \frac{\partial T_{s}}{\partial z}=-h_{f s} \cdot\left(T_{f}-T_{s}\right), \text { at } z=d_{f} \\
K_{s} \cdot \frac{\partial T_{s}}{\partial z}=-h_{s c} \cdot T_{s}, \text { at } z=d_{f}+d_{s}
\end{gathered}
$$

where $h_{f s} \equiv 1 / R_{f s}$ is the thernal boundary conductance at the film/substrate interface, $h_{s c} \equiv 1 / R_{s c}$ the thermal boundary conductance at the substrate/heat-sink interface, $d_{s}$ the thickness of the substrate, and $R_{f s}, R_{s c}$ represent the thermal boundary resistances of the film/substrate interface and of the substrate/heat-sink interface, respectively.

Using a complex sinusoidal modulation treatment similar to (8), with the introduction of a chopping angular frequency $\omega=2 \pi \cdot f$, the heat conduction equations of (9) and (10) can be reduced into complex time-independent heat conduction equations, and the solution of $\left.T_{f}(z, t) \equiv T_{f 0}(z) \cdot \exp (-i \omega) \cdot t\right)$ can be exactly solved, where $T_{f 0}(z)$ is a complex quantity, representing the complex temperature rise of the film at a depth in the range $0<z<d_{f}$. The depth-average of $T_{f o}(z)$ can, then, be deduced as expressed by

$$
\left\langle T_{f 0}\right\rangle \equiv \frac{1}{d_{f}} \cdot \int_{0}^{d_{f 0}} T_{f}(z) \equiv\left\langle\bar{T}_{f}\right\rangle \cdot T_{0}
$$


where $\quad T_{0} \equiv \frac{d_{f}^{2}}{K_{f}} \cdot \Im_{0} \cdot \frac{1-R_{f}}{\mu_{r}} \cdot \beta_{f} \cdot \tau_{w} \cdot \tau_{c}$,

$$
\begin{gathered}
\left\langle\bar{T}_{f}\right\rangle \equiv \bar{A} \cdot\left\{\frac{\bar{H}+\frac{1}{\bar{B}_{f s}}+\left(\frac{\bar{\gamma} \bar{G}}{\bar{F}}\right) \cdot\left[1-\exp \left(-\bar{\beta}_{f}\right)\right] \cdot \bar{C}}{\bar{\gamma} \cdot \bar{G} \cdot \bar{F} \cdot \bar{C}+\frac{\bar{F}^{2}}{\bar{B}_{f s}}+\bar{F} \cdot \operatorname{coth}(\bar{F})}-\bar{D}\right\}, \\
\bar{A} \equiv \frac{\bar{\beta}_{f}}{\left(\bar{\beta}_{f}+\bar{F}\right) \cdot\left(\bar{\beta}_{f}-\bar{F}\right)},
\end{gathered}
$$$$
\bar{C} \equiv \frac{\left(\bar{G} \cdot \bar{F} \cdot \bar{E}+\bar{B}_{s c}\right) \cdot \tanh (\bar{G} \cdot \bar{F})-\left(\bar{G} \cdot \bar{F}+\bar{B}_{s c} \cdot \bar{E}\right)}{\left(\bar{G} \cdot \bar{F}+\bar{B}_{s c} \cdot \bar{E}\right) \cdot \tanh (\bar{G} \cdot \bar{F})-\left(\bar{G} \cdot \bar{F} \cdot \bar{E}+\bar{B}_{s c}\right)}
$$$$
\bar{D} \equiv \frac{1-\exp \left(-\bar{\beta}_{f}\right)}{\bar{\beta}_{f}^{2}},
$$$$
\bar{E} \equiv \tanh \left[\bar{G} \cdot \bar{F} \cdot\left(1+\frac{d_{s}}{d_{f}}\right)\right] \text {, }
$$$$
\bar{F} \equiv \sqrt{-i \cdot 2 \pi \cdot f \cdot \frac{d_{f}^{2} \cdot \rho_{f} \cdot c_{f}}{K_{f}}},
$$$$
\bar{H} \equiv \frac{\cosh (\bar{F})-1}{\bar{F} \cdot \sinh (\bar{F})}+\left(\frac{1}{\bar{\beta}_{f}}-\frac{1}{\bar{B}_{f S}}\right) \cdot \exp \left(-\bar{\beta}_{f}\right),
$$

where the overline symbol is assigned to artificially-defined dimensionless quantities, $i \equiv \sqrt{-1} \quad, \quad \overline{\boldsymbol{\beta}}_{f} \equiv \boldsymbol{\beta}_{f} \cdot d_{f}$, $\bar{\gamma} \equiv\left(\rho_{f} \cdot c_{f}\right) /\left(\rho_{s} \cdot c_{s}\right), \bar{G} \equiv \sqrt{\left(\rho_{s} \cdot c_{s} \cdot K_{f}\right) /\left(\rho_{f} \cdot c_{f} \cdot K_{s}\right)}$, $\bar{B}_{f s} \equiv\left(h_{f s} \cdot d_{f}\right) / K_{f}$ and $\bar{B}_{s c} \equiv\left(h_{s c} \cdot d_{f}\right) / K_{s}$.

The amplitude of the sinusoidal temperature rise of the film is related to the modulus of the complex quantity $\left\langle T_{f 0}\right\rangle$. The bolometric optical response of the film is, thus, given by

$$
V_{o r}=\frac{1}{\sqrt{2}} \cdot I \cdot \frac{d R}{d T} \cdot\left|\left\langle T_{f 0}\right\rangle\right|,
$$

where the factor $1 / \sqrt{2}$ is due to the measurement technique using a lock-in amplifier, and $R$ the resistance of the film measured at the film temperature $T$.

\section{The Case of Strong Thermal Coupling}

In order to compare (23) with experimental results, simplified conditions are required in an intermediate chopping frequency range, restricted by $\bar{\beta}_{f} \gg 1,|\bar{F}| \ll<1$, and $\bar{G} \cdot|\bar{F}| \cdot\left[1+\left(d_{s} / d_{f}\right)\right] \gg>1$ such that (23) can be reduced into a relatively useful form, expressed by

$V_{o r}=\frac{I}{\sqrt{2}} \cdot \frac{d R}{d T} \cdot\left[\mathfrak{I}_{0} \frac{\left(1-R_{f}\right)}{\mu_{r}} \tau_{w} \tau_{c}\right] \frac{\bar{\Gamma}(f)}{\sqrt{2 \pi \rho_{s} c_{s} K_{s} \cdot f}}$

where

$$
\begin{gathered}
\bar{\Gamma}(f) \equiv\left(\frac{1+U_{1} \cdot \sqrt{2 f}+U_{1}^{2} \cdot f}{1+U_{2} \cdot \sqrt{2 f}+U_{2}^{2} \cdot f}\right)^{\frac{1}{2}}, \\
U_{1} \equiv \sqrt{2 \pi \cdot \rho_{s} \cdot c_{s} \cdot K_{s}} \cdot\left(\frac{1}{h_{f s}}+\frac{d_{f}}{2 K_{f}}\right), \\
U_{2} \equiv \frac{2 \pi \cdot \rho_{f} \cdot c_{f} \cdot d_{f}}{\sqrt{2 \pi \cdot \rho_{s} \cdot c_{s} \cdot K_{s}}} .
\end{gathered}
$$

Equation (24) provides a very similar form to that given by a strong thermal-coupling model suggested by Dwir and Pavuna [2]. The only difference is that in their model the factor $\bar{\Gamma}(f)$ corresponds to unity, whereas in our model a more general expression is derived, including the influences of $d_{f}$ and $h_{f s}$. The expression in (24) can, thus, be used to explain why some $V_{o r}$ results of a group follow $f^{-0.5}$ dependence, whereas others do not. In fact, the $\bar{\Gamma}(f)$ values of Dwir and Pavuna's results is found to occur in the range of $1.0024 \leq \bar{\Gamma}(f) \leq 1.0784$, which is indeed very close to unity in a relatively wide chopping frequency range. The frequency-dependent parameter $\bar{\Gamma}(f)$ is, thus, defined as the film factor of the system.

\section{The Case of Weak Thermal Coupling}

The optical response with weak thermal-coupling to the heat-sink has been demonstrated by Dwir and Pavuna [2], where the substrate was virtually isolated to the heat-sink, using an insulating support. To explain the chopping frequency dependence of the weak coupling response, the value of $\bar{B}_{s c}$ is assumed to be 'negligibly small but nonzero' in order to prevent the unreasonably divergence of $V_{o r}$ at the zero frequency limit, as well as requiring $\bar{\beta}_{f} \gg 1$ and $\bar{G} \cdot|\bar{F}| \cdot\left[1+\left(d_{s} / d_{f}\right)\right] \ll 1 \quad$. Equation (23) can, therefore, be reduced into a simpler expression given by

$$
\begin{aligned}
V_{o r}=I \cdot \frac{d R}{d T} \cdot\left[\frac{\left(1-R_{f}\right)}{\sqrt{2} \mu_{r}} \cdot \tau_{w} \cdot \tau_{c}\right] \\
\frac{P_{f}}{2 \pi \cdot\left[A_{f} \cdot\left(\rho_{f} c_{f} d_{f}+\rho_{s} c_{s} d_{s}\right)\right]} \cdot \frac{1}{f}
\end{aligned}
$$


where $\mathfrak{I}_{0} \equiv P_{f} / A_{f}, A_{f}$ is the irradiated area of the superconducting pattern, $P_{f}$ is the irradiated laser power on the film surface. The $f^{-1}$ dependence of the response is consistent with the results of Dwir and Pavuna [2].

The weak coupling response was interpreted by a classical bolometric model [2], where the bolometer is modeled as a single bulk thermal mass (or thermal capacitance) $C=\rho_{s} \cdot c_{s} \cdot d_{s} \cdot A_{f}$, connected to the heat-sink by a thermal link with thermal conductance $G$, absorbing the irradiated power $P_{f}$ on the film surface with an optical absorption efficiency $0 \leq \eta \leq 1$. The characteristic time constant of the response is defined by $\tau \equiv C / G$, where $V_{\text {or }}$ is expressed by

$$
V_{o r}=I \cdot \frac{d R}{d T} \cdot \frac{\eta \cdot P_{f}}{G \cdot \sqrt{1+(2 \pi \cdot f \cdot \tau)^{2}}}
$$

The expression in (29) is compared to both (23) and (28) at the limit of zero chopping frequency as well as under the condition of $2 \pi \cdot f \cdot \tau \gg>1$, such that explicit expressions of $C, G, \tau$, and $\eta$ can be derived as expressed by

$$
\begin{gathered}
C=A_{f} \cdot\left(\rho_{f} \cdot c_{f} \cdot d_{f}+\rho_{s} \cdot c_{s} \cdot d_{s}\right) \\
G=A_{f} \cdot\left(\frac{d_{f}}{2 K_{f}}+\frac{1}{h_{f}}+\frac{d_{s}}{K_{s}}+\frac{1}{h_{s c}}\right)^{-1}, \\
\tau=\left(\rho_{f} c_{f} d_{f}+\rho_{s} c_{s} d_{s}\right) \cdot\left(\frac{d_{f}}{2 K_{f}}+\frac{1}{h_{f s}}+\frac{d_{s}}{K_{s}}+\frac{1}{h_{s c}}\right), \\
\eta=\frac{\left(1-R{ }_{f}\right)}{\sqrt{2} \mu_{r}} \cdot \tau_{w} \cdot \tau_{c} .
\end{gathered}
$$

It is found that the expression of the total thermal mass of the system in (30) includes both the thermal mass of the film as well as the thermal mass of the substrate. The result in (31) indicates that the total thermal resistance of the system is a summation of individual thermal resistances in a seriesconnected heat conduction circuit, where the factor $1 / 2$ of the film is caused by the average procedure in (14). If the Jouleheating effect is taken into account [8], a more general expression of the thermal conductance $G_{e}$ is given by

$G_{e}=A_{f} \cdot\left(\frac{d_{f}}{2 K_{f}}+\frac{1}{h_{f s}}+\frac{d_{s}}{K_{s}}+\frac{1}{h_{s c}}\right)^{-1}-I^{2} \cdot \frac{d R}{d T}$

The Joule-heating effect in (34) can be ignored, so that $G_{e} \cong G$, if the bias current $I$ is sufficiently small.

\section{CONCLUSION}

The modified heat-conduction model is found to be capable of being applied to both the weak and strong thermal-coupling cases of the bolometric response of superconducting films. In the strong coupling case, the optical response is simplified for a specific intermediate chopping frequency range, so that the formulation of the response is very similar to that derived from a simple semi-infinite heat-conduction system, except that a frequency dependent film factor is introduced. The model can also be used to describe the results of weak coupling systems in a more general sense, where a $f^{-1}$ dependence of the response is deduced. By comparing the derived results with a simple bolometric model, general expressions of the thermal conductance $G$, thermal capacitance $C$, radiation absorption efficiency $\eta$ and response time constant $\tau$ of the bolometric system have been obtained, where the influences of the film, substrate and the thermal boundaries are all considered. The modified model is, thus, capable of unifying both the weak and strong coupling cases, which previously required two different models. However, the model is only suitable to be applied to optically thick films. If the film surface is smooth, a more general expression of the response can further be deduced for optically thin films.

\section{ACKNOWLEDGMENT}

The authors wish to thank Dr. C.R.M. Grovenor, Dr. M.J. Goringe, Dr. A. Guldeste, Dr. S. Aslam, Dr. H.C. Lai, Dr. B. Minakovic, and Dr. J.R. Waldram for their valuable advice and assistance.

\section{REFERENCES}

[1] J.D. Chern, H.C. Lai, A. Guldeste, S Aslam, D.H.T. Wu, D. Dew-Hughes, C.R.M. Grovenor, M.J. Goringe, and B. Minakovic, IEEE Trans. Appl. Supercond., vol. 3(1), pp. 2128-2131, 1993.

[2] B. Dwir, and D. Pavuna, J. Appl. Phys., vol. 72(9), pp. 3855-3861, 1992.

[3] C.J. Haung, C.Y. Chang, and T.Y. Tseng, J. Appl. Phys, vol. 72(12), pp. 5786-5791, 1992.

[4] W. Eidelloth, IEEE Trans. Magn., vol. 27(2), pp. 28282831, 1991.

[5] Z.D. Genchev, R.A. Chakalov, J.D. Chern, and D. DewHughes, Supercond. Sci. Technol. vol. 9, pp. 523-528, 1996.

[6] J.H. Hao, F.Q. Zhou, X.R. Zhao, H.D. Sun, X.J. Yi, and Z.G. Li, IEEE Trans. Appl. Supercond,, vol. 3(1), pp. 2167-2169, 1993.

[7] M.I. Flik, P.E. Phelan, and C.L. Tien, Cryogenics, vol. 30, pp. 1118-1128, 1990.

[8] J. Clarke, G.I. Hoffer, P.L. Richards, and N.-H. Yeh, J. Appl. Phys., vol. 48(12), pp. 4865-4879, 1977. 